Ploog, K., Cance, C., Badin, F. \& Nous, C. (à paraître en 2021). Figures et construction du soin dans le parcours de soin pluridisciplinaire, Études de Linguistique Appliquée ELA, numéro spécial « Perspectives D'interventions En Linguistique Appliquée: Quelles Réponses Face Aux Besoins Sociétaux ».

\title{
FIGURES ET CONSTRUCTION DU SOIN DANS LE PARCOURS DE SOIN PLURIDISCIPLINAIRE
}

Sur la base de deux corpus d'interactions de soin, recueillis en santé mentale et en addictologie, cet article retrace l'élaboration de deux profils d'usageres en proposant une analyse qualitative des extraits d'interactions correspondants. Nous mettons en exergue une ressource de cette élaboration collective par l'équipe pluridisciplinaire: les figures, objets émergents, constitués intersubjectivement, qui apparaissent dans des fonctions d'expansion et de condensation, liées à la construction du soin dans le parcours qui l'intègre.

\section{INTRODUCTION : LE PARCOURS DE SOIN EN EQUIPE PLURIDISCIPLINAIRE}

Les travaux en sociologie, sociolinguistique interactionnelle, analyse conversationnelle et analyse du discours ont mis en exergue la part langagière du soin (Heritage \& Maynard, 2006 ; Gülich, 2012; Traverso, 2017 ; Gajo, 2004 ; Piot, 2018, Ploog, Mariani-Rousset \& Equoy-Hutin, 2018 et Garric \& Herbland, 2020 ; parmi d'autres). Bien plus qu'un acte ou ensemble d'actes quantifiables en durée ou efficience (tel le référentiel de l'ARS) le soin doit être compris comme relation humaine où les participants s'engagent à "prendre soin" ; au centre de cette coopération se trouve non pas une pathologie, mais une personne, participant activement (en opposition à l'appellation commune, inadéquate de "patient") à l'élaboration du soin. La construction du soin est donc un processus collaboratif complexe, négocié entre différents acteurs.

Dans cette perspective, les échanges entre professionnel.les d'une équipe pluridisciplinaire constituent une élaboration à plusieurs voix du profil (pathologique mais aussi psychologique et social) du "patient", nommé ici usager.e pour les raisons évoquées plus haut. Les soignant.es issus de spécialités diverses partagent en effet les informations pour objectiver et enrichir leur compréhension du profil dans le but de mettre au point collectivement une stratégie de soin, développée ensuite de façon fractionnée en actes individuels de différentes spécialités, complémentaires et coordonnés. Aussi, la construction discursive du profil de l'usager.e apparaît comme « méthode » du soin.

Nous nous proposons d'analyser ce processus collaboratif dans une temporalité médiane, au-delà de l'interaction de soin isolée. La réflexion présentée se veut être une contribution de la linguistique appliquée au champ d'intervention du soin: la connaissance fine des ressources 
interactionnelles permettra de proposer, à terme, des remédiations, sous la forme par exemple de modules de formation professionnelle médicale et paramédicale à la communication en situation de soin.

\section{DONNÉES D'ÉTUDE : CORPUS LONGITUDINAUX EN SOIN NON SOMATIQUE}

Notre réflexion est basée sur deux corpus longitudinaux d'interactions de soin recueillis en structure pluridisciplinaire, «Daumezon Dispo Ados » (Cance) et « Parole émergée » (Ploog, Equoy-Hutin \& MarianiRousset, 2014).

Le dispositif de soins pour Adolescents de ' $^{\prime}$ PSSM $^{1}$ Georges Daumezon (Fleury les Aubrais, 45, DDA dans la suite) accueille des adolescent.es présentant différents types de troubles (du comportement, anxieux, états limite, parmi d'autres). La prise en charge globale s'articule dans un parcours de soin sur une durée courte (séjours de crise), le cas échéant prolongée par une hospitalisation de jour ou un nouveau séjour, ponctuée par des entretiens médicaux et psychologiques de suivi et de bilan. Le dispositif propose en outre des activités thérapeutiques variées qui participent au soin, comme l'art-thérapie, la relaxation, ou le photolangage, ainsi qu'un suivi scolaire. Le soin est construit en articulation avec les activités de la vie quotidienne à l'hôpital (repas, temps calme, tv, lever, coucher). Le corpus est constitué de $30 \mathrm{~h}$ d'interactions de soin et de suivi entre professionnels.

Le corpus « Parole émergée » (PE dans la suite) a été recueilli au CSAPA ${ }^{2}$ L'Escale-ANPAA70 (Vesoul, 70) durant plus d'une année ${ }^{3}$. L'établissement accueille les personnes en situation d'addiction sans condition préalable mais exige l'engagement dans un projet construit avec l'équipe, qui devra se réaliser au fil du parcours de soin. Le temps de prise en charge par la structure varie en fonction du projet et en fonction de sa réalisation effective. La prise en charge globale est évolutive en fonction de l'émergence des objets de soin, et déclinée en suivi par différentes spécialités médicales et paramédicales. La coordination des prises en charges est assurée lors de la réunion de service hebdomadaire.

Ces deux terrains, malgré leurs spécificités, présentent des convergences importantes. Il s'agit tout d'abord de types de soin principalement non somatiques et fractionnés sur des temps (individuels et collectifs) successifs, qui représentent des interactions avec plusieur.e.s professionnel.les de différentes spécialités médicales (psychiatre, médecin

Établissement Public en Santé Mentale.

Centre de Soins, d'Accompagnement et de Prévention en Addictologie.

Métadonnées publiées sous : https://cocoon.huma-num.fr/exist/crdo. 
généraliste), paramédicales (infirmier.e, aide-soignant.e) et socioéducatives (éducateur.ice, assistant.e social.e, aide-médicopsychologique). Les deux contextes supposent une coordination soutenue entre les membres de l'équipe qui axent la prise en charge sur une formulation du projet de soin $^{4}$ avec l'usager.e et son environnement. Pour cette contribution, nous avons choisi d'observer les ressources langagières liées à la construction des objets du soin ${ }^{5}$ mobilisées par les équipes professionnelles dans différentes temporalités :

- dans une temporalité courte, les interactions de soin observées (corpus $\mathrm{PE}$ ), presque toujours dyadiques (entre usager.e et soignant.e) et très récurrentes. Elles comportent des contenus spécifiques prévus par le protocole (ici test d'urine, délivrance de traitement, point sur la santé générale); cependant, la présence de thématiques non liées au protocole est fréquente et le temps accordé aux différents volets du protocole reste très variable (Cance \& Ploog, 2020).

- dans une temporalité intermédiaire, les transmissions (corpus DDA) constituant les trois passages de relais par $24 \mathrm{~h}$ entre équipes soignantes. Le déroulement et les thématiques varient selon le moment de la journée mais possèdent un caractère très routinier où les rôles de chacun sont en outre précisément définis ; il s'agit de passer en revue les usager.es (ici des adolescent $\cdot$ es) par ordre de chambre.

- la temporalité médiane, la plus longue prise en compte, dans cette étude est représentée par les réunions d'équipe (corpus $\mathrm{PE}$ ), à fréquence hebdomadaire. Elles sont pilotées par le chef de service (adjoint de la direction), ou, en l'absence de celui-ci, par la médecin coordinatrice. Le déroulement en est plus ou moins standard, bien que le temps alloué aux différents volets dépende de l'actualité.

Ces trois temporalités interactionnelles du soin entrent en résonance avec la temporalité du soin lui-même et de la connaissance de la personne en situation de soin sur des mois voire sur de nombreuses années. Enfin, bien que les analyses ci-après focalisent sur la part verbale du processus, il est important de noter le caractère multimodal de ces interactions, le déroulement s'appuyant sur les formulaires du dossier papier du patient, sur la rédaction de l'ordonnance, ou encore sur le suivi de patient via la base de données informatique.

\section{APPROCHE : TRACER LES OBJETS DU SOIN}

4 Cadrage global et collectif de la façon de «prendre soin » de la personne à partir de l'identification de ses besoins.

Éléments concrets sur lesquels porte le soin tant au niveau somatique que psychologique ou socio-éducatif. 
Il s'agira d'observer les procédés de construction des objets constitutifs du soin par le "travail de formulation" (Gülich \& Mondada, 2001 ; Dausendschön, Gülich \& Krafft, 2007), : les processus de formulation de la pensée et de l'expérience sensible posent en effet de nombreux défis cognitifs, linguistiques et conversationnels aux interlocuteurs. L'effort consacré à cette élaboration collaborative laisse des traces dans le discours, qui ont été saisies en analyse conversationnelle comme des pratiques de monstration par lesquelles les partenaires de l'interaction rendent manifeste le travail cognitif et communicatif fourni en temps réel. Les personnes en situation de soin (usager.es) se distinguent par des vulnérabilités ${ }^{6}$ accentuées, singulières et souvent multiples qui peuvent avoir trait à l'autonomie, un fonctionnement familial, des comportements déviants, des problèmes communicationnels, ou encore des comorbidités. En interaction, la mise en mots du profil de l'usager.e s'opère notamment par la verbalisation d'éléments factuels illustrant ces vulnérabilités, que nous qualifierons d'attributs (au sens général : prédicats), plus ou moins connus par les membres de l'équipe, construits lors des réunions (p.ex. elle est très sarcastique, DDA; il vit au jour le jour, PE). Sur fond d'inscriptions disciplinaires variées, personne ne dispose de l'ensemble des attributs relatifs à un.e usager.e. Parmi les attributs qui caractérisent l'usager.e émergent des figures. D'inspiration gestaltiste, ce concept repris par les grammaires cognitives (Langacker 1986), permet ici de saisir des objets discursifs plus ou moins stabilisés - émergeant en interaction, donc, intersubjectifs et dynamiques - qui élaborent les représentations collectives de l'équipe dans la construction du parcours de soin autour de l'usager.e.

Parmi d'autres figures possibles, il s'agira ici d'observer l'émergence des figures lexico-référentielles à travers l'analyse des ressources interactionnelles mises en œuvre. La démarche, inspirée de l'analyse du discours et de l'analyse conversationnelle, consiste à relever dans l'interaction les attributs caractérisant l'usager.e pour ensuite rechercher, dans une approche séquentielle, les motifs langagiers particuliers qui émergent au fil du traitement conversationnel effectué par les interactants, à partir de la mise en mots (Gülich \& Mondada 2001) ; les nombreuses reformulations (répétitions, re-thématisations) observées au fil des échanges conduisent à envisager ces élaborations d'attributs comme figures émergeant d'un véritable travail de formulation conjoint. L'exposition de ces mécanismes permet d'en comprendre le rôle dans le processus du soin et de valoriser le temps qui y est consacré par l'équipe.

6 La définissant comme " potentialité à être blessé », Soulet remarque qu'elle renvoie à la fois à «un déficit de ressources ou (...) de conditions cadres affectant la capacité individuelle à faire face à un contexte critique ", et à la " capacité de saisir des opportunités (...) pour surmonter cette épreuve » (Soulet, $2014: 63$ ). 
Nous focalisons cette contribution sur l'élaboration de deux profils d'usager.es, à travers une micro-analyse qualitative de plusieurs extraits d'interactions (représentant les différentes temporalités présentées sous 2.2) rendant compte des parcours de soin fractionnés.

\section{3. ÉTUDES DE CAS}

\subsection{Triche à la crapette (DDA)}

Pauline $^{7}$ est arrivée dans l'unité de soins quelques jours auparavant. L'adolescente de 15 ans et l'équipe sont en phase d'observation réciproque, ce que la série des trois transmissions sous étude permet de retracer en partie. Les interactions analysées se caractérisent par une temporalité intermédiaire (sur moins de $24 \mathrm{~h}$ ). Au delà des échanges d'information concernant le suivi en cours et à venir, les réunions ont pour objet principal d'essayer de caractériser Pauline afin de construire un projet de soin adapté. Perçue par l'équipe comme une jeune fille très autoritaire, qui peine à trouver sa place, elle semble avoir "un contact un peu particulier".

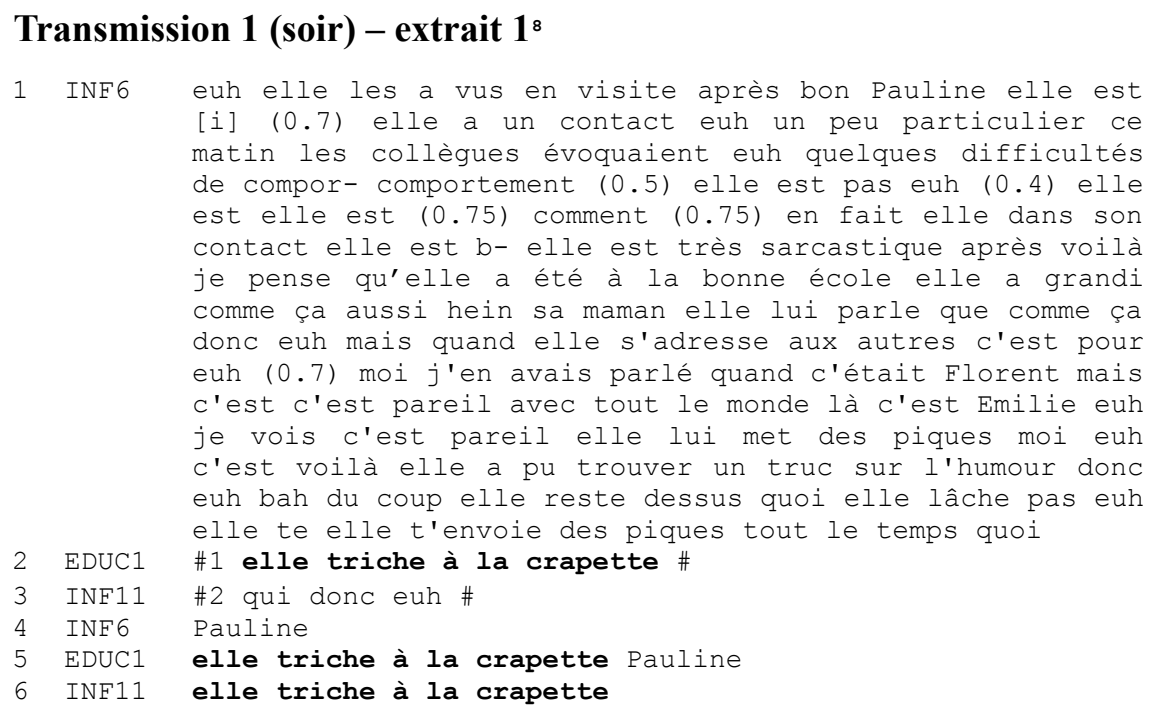

Lors de la première réunion de transmission étudiée (entre équipes de l'après-midi et de nuit), l'infirmière en charge des transmissions (INF6) donne d'abord des informations suite au rendez-vous entre les parents et la psychiatre (1.1). Elle opère ensuite une transition thématique pour ébaucher une caractérisation du comportement de Pauline à travers des

7 Les prénoms des usager.es ont été modifiés et les autres données identifiantes anonymisées.

8 Conventions de transcription : \# : paroles $(1,2)$ chevauchées ; chiffres entre parenthèses : durée de pauses; caractères gras : construction des figures. 
attributs successifs (contact particulier, très sarcastique, envoie des piques) ponctuée de nombreuses pauses et modalisations, indiquant une élaboration progressive, non stabilisée que l'infirmière propose à ses collègues (1.1). A la fin de cette longue caractérisation, l'éducatrice (EDUC1) introduit un nouvel attribut, qui décrit un comportement observé en instance : elle triche à la crapette (1.2), attribut à la fois en continuité avec le tour précédent (comme contribution à la liste d'attributs) et en rupture sur le plan formel. Après avoir été répété par l'éducatrice (1.5), l'attribut est repris par une infirmière (INF11) (1.6), ce qui permet son émergence comme figure.

La figure est ensuite étayée collectivement, par EDUC1 et INF11 (extrait 2) à travers une reprise en écho (ratification : 1.2-3) et des étayages conjoints (reformulations : 1.4) :

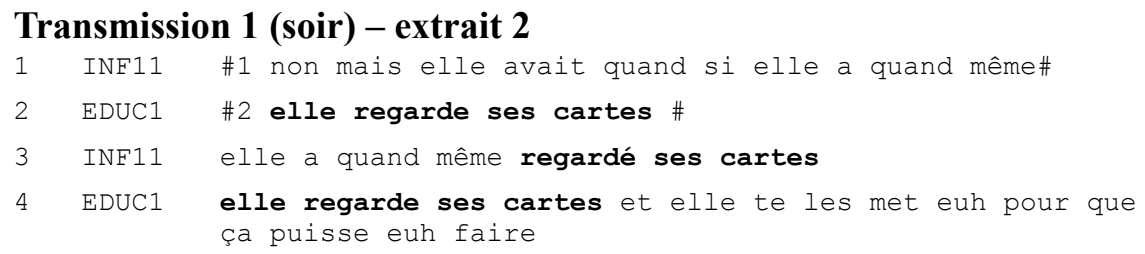

L'échange débouche sur une généralisation initiée par INF6 et coconstruite avec EDUC1 (extrait 3), qui élabore le rapport aux autres problématiques de l'usagère (1.1-7). EDUC1 remobilise enfin la figure de la crapette en passant de tricher à gagner (1.8) :

\section{Transmission 1 (soir) - extrait 3}

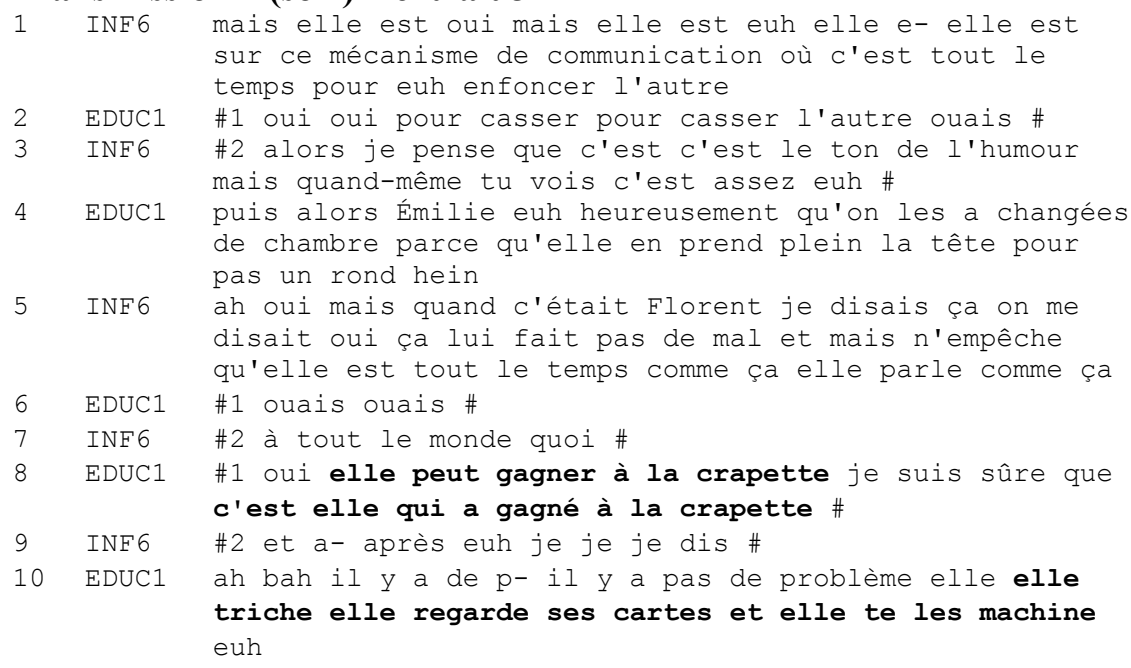

La transmission suivante (matin), centrée sur le suivi et le mode de fonctionnement de Pauline, est concise, la figure de la crapette n'y est pas 
présente. Lors de la dernière des trois transmissions analysées, entre équipes matin et après-midi, INF2 commence par rappeler le suivi en cours et à venir et informe sur l'activité actuelle de Pauline (1.1-4):

\section{Transmission 3 (après-midi) - extrait}

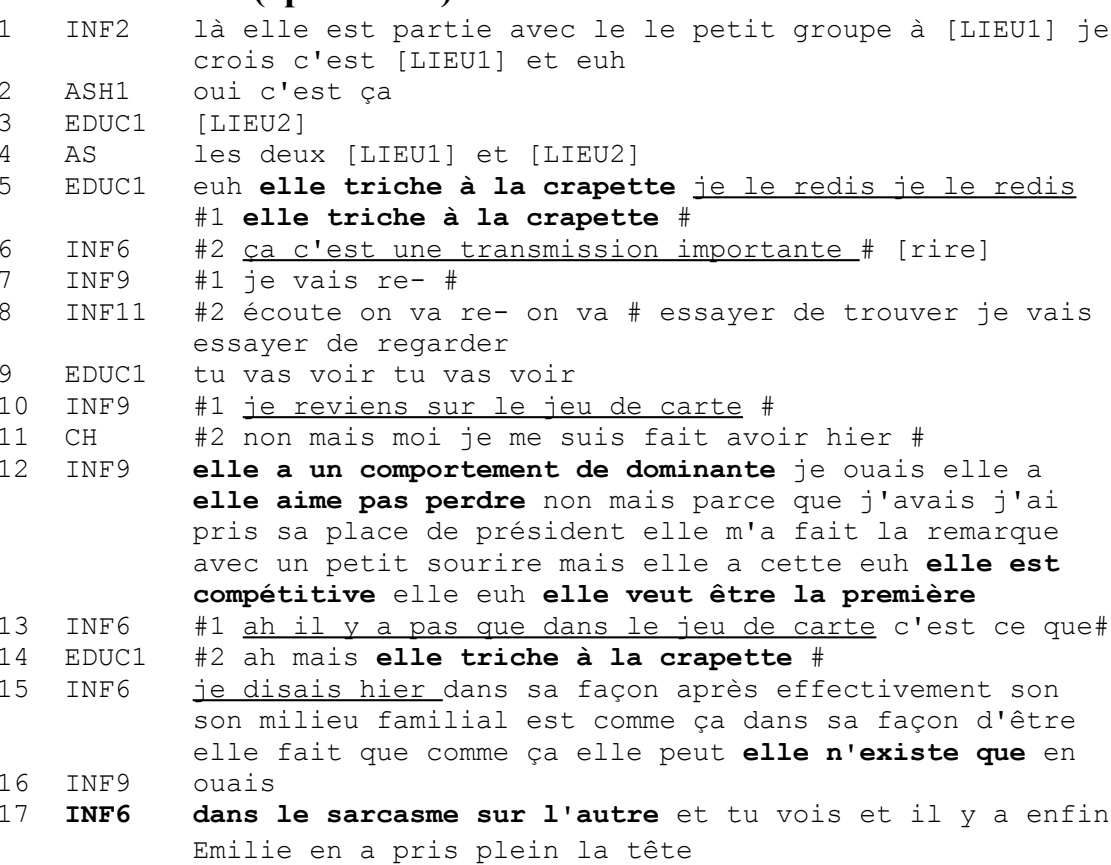

EDUC1 va rebondir (1.5) en remobilisant la figure introduite la veille par une annonce théatralisée, "radiophonique", doublée d'un commentaire métadiscursif ${ }^{9}$ (je le redis je le redis). Cette intervention suscite un nouveau commentaire métadiscursif de INF6 (en charge des transmissions la veille au soir) qui questionne l'enjeu de cette information (1.6 : ça c'est une transmission importante). Or, la figure sert à nouveau et de façon plus claire de support pour élaborer le profil de Pauline : INF9, non présente la veille, prend appui sur l'exemple de la crapette pour rapporter ce comportement à d'autres jeux de cartes (elle a un comportement de dominante / elle aime pas perdre / elle est compétitive / elle veut être la première; 1.12). Alors INF6 généralise l'observation au comportement global de l'usagère (dans sa façon d'être elle fait que comme ça elle n'existe que en dans le sarcasme sur l'autre ; 1.13-17).

La figure prend ici un rôle majeur dans la construction intersubjective du profil de l'adolescente. La caractérisation progressive de Pauline par les soignantes procède par énumération d'attributs, formulés comme qualifications ou comme comportements observés. Dans ce contexte, les professionnel.les élaborent en moins de 24 heures la figure "triche à la

9 souligné dans l'extrait. 
crapette" en prenant appui sur elle pour construire une représentation collective du profil de l'adolescente. Nous qualifions ce processus d'émergence et de négociation d'une représentation commune en interaction comme expansion, qui semble constituer une fonction élémentaire des figures interactionnelles: l'observation d'un comportement singulier (triche à la crapette) réitéré, est étendue aux jeux de cartes pour ensuite donner lieu, en lien avec d'autres éléments du comportement de Pauline, à la projection d'un fonctionnement général (de domination), à partir duquel, l'équipe va élaborer une stratégie de soins (relationnels surtout) pour travailler avec la jeune fille sur cette problématique.

\subsection{En claquettes (PE)}

Fabrice est suivi par le CSAPA depuis 15 ans. Beaucoup de pistes ont été envisagées et testées (sans grand succès) pour lui permettre de prendre pied dans la vie sociale. Les interactions entre septembre et décembre 2013, faisant l'objet des analyses des pages suivantes, thématisent l'engagement de l'usager dans son projet. En septembre, l'usager a mûri (avec l'équipe) un projet de postcure, pour lequel la condition est l'arrêt complet préalable de la consommation de drogue ( ?), programmé en hôpital psychiatrique. Coïncidence ou non, le premier jour de la cure, le juge d'application des peines se présente au domicile de sa mère pour annoncer l'exécution imminente d'une peine de prison. La situation est confuse entre le discours de l'usager, celui de la mère, et les informations obtenues par les membres de l'équipe auprès de différents interlocuteurs d'autres structures d'aide.

Le "partenariat" de soin entre Fabrice et les membres de l'équipe pluridisciplinaire est marqué par une relation très asymétrique, avec des échanges courts, complémentaires, quasi-transactionnels, dans les interactions de soin: dans l'interaction entre Fabrice et la médecin, recueillie quelques jours avant son départ en hôpital psychiatrique, l'ensemble du protocole est déroulé en $4 \mathrm{mn} 15 \mathrm{sec}$. En somme, Fabrice se montre toujours coopératif, mais semble perdu entre les interlocuteurs et les rendez-vous de son parcours de soin.

Entre septembre et décembre, les soignant.es échangent à plusieurs reprises pour comprendre les tenants et aboutissants de la situation globale de l'usager (projet de cure, mise à l'épreuve judiciaire, manque d'autonomie), mais celui-ci reste fuyant, en n'honorant pas les rendezvous et en fournissant des explications qui ne résistent pas aux vérifications...

Phrase de transition pour introduire l'extrait 


\section{8b_mailles (13/10)}

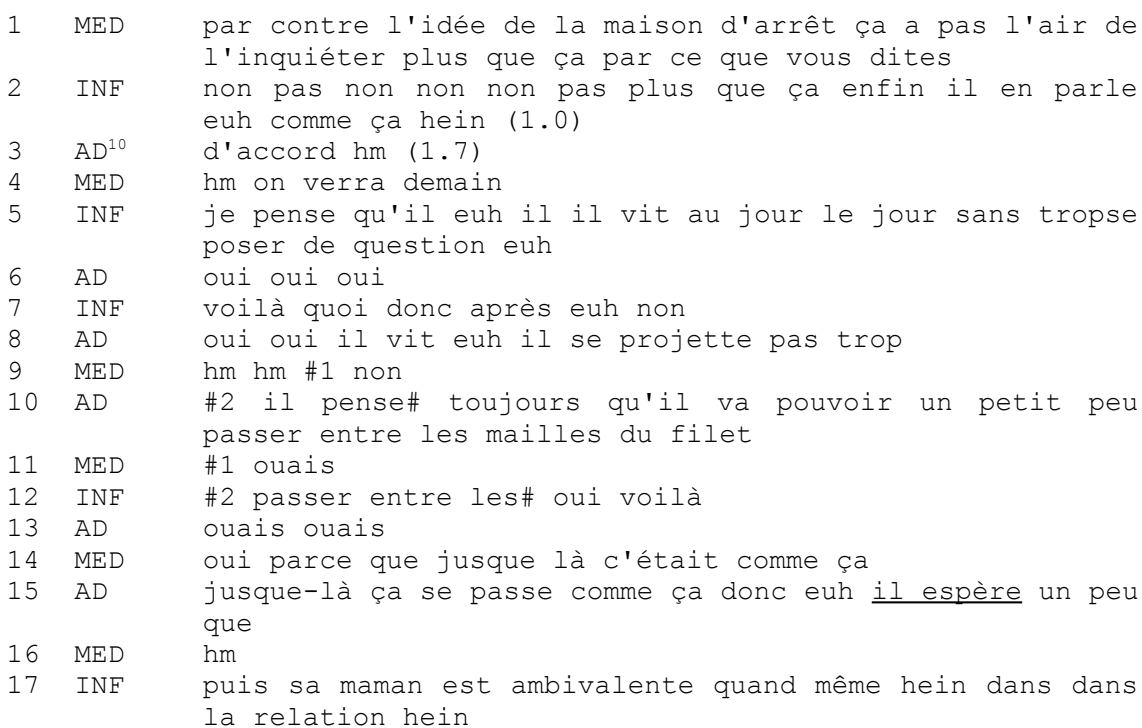

Différents attributs sont formulés : ça a pas l'air de l'inquiéter plus que ça; il vit au jour le jour, il se projette pas trop, il pense pouvoir passer entre les mailles $d u$ filet. L'on note, d'abord, que l'élaboration discursive de la perplexité est collective, les attributs sont construits conjointement avec des répétitions (1.10/12) ou des reformulations (1.5/8 ; $1.14 / 15)$; puis, que les co-constructions marquent les rapports de place : en effet, les répétitions, ici des ratifications de l'attribut, sont généralement réalisées par l'infirmière (INF), alors que les reformulations, étayages de l'attribut, sont à l'initiative du cadre (AD). La représentation commune est l'absence d'engagement de l'usager dans son projet. L'équipe en retient que "Fabrice lui il veut pas grand chose", comme le résume un soignant à un autre moment.

L'expression de la stupéfaction alterne avec les stratagèmes pour trouver une "prise" sur l'usager. L'élaboration des attributs recourt alors au récit (1.7-15), qui se termine par un discours animé (en italiques) :

\section{PE350b_claquettes (3/12)}

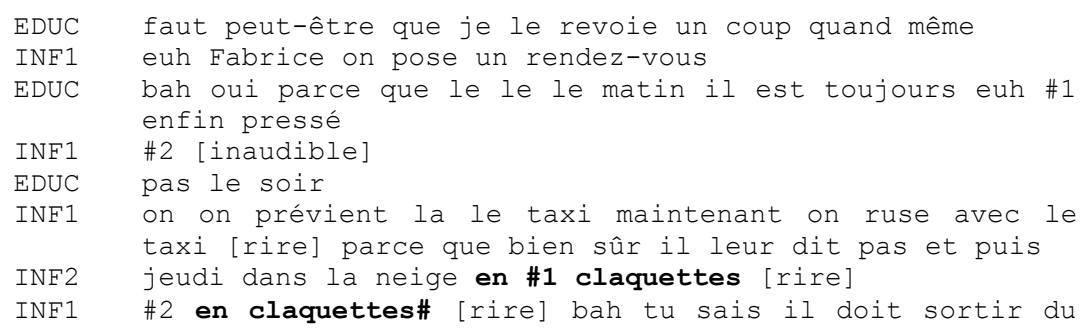

10 AD : adjoint de direction. 


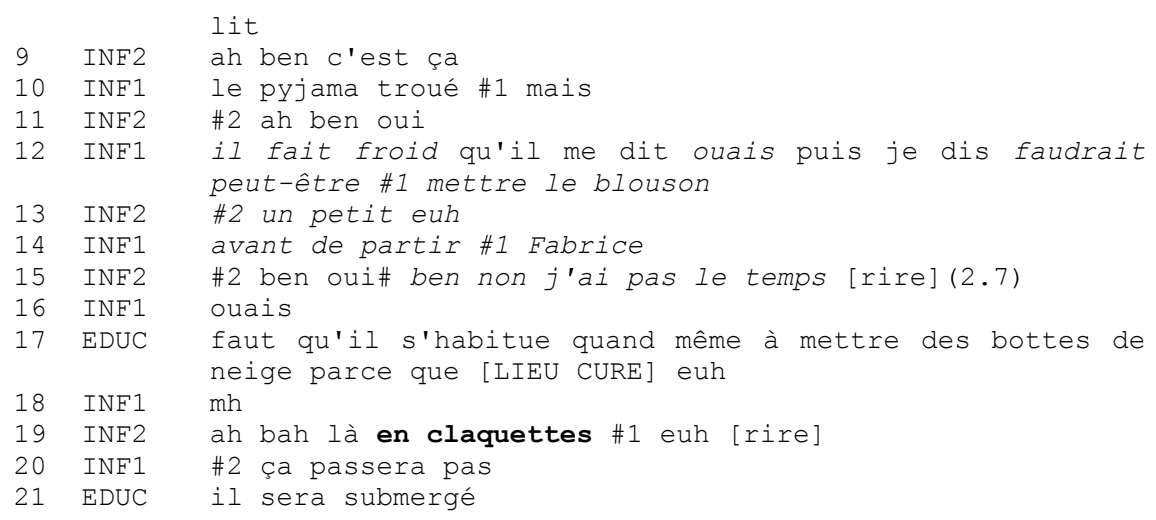

Dans ce contexte, la figure des claquettes fédère les représentations de l'équipe : ce n'est pas comme ça qu'il pourra aller de l'avant. L'attribut est introduit avec un effet dramatique, par une phrase segmentée (1.7: jeudi / dans la neige / en claquettes [rire]). La figure est co-élaborée de façon presque synchrone par l'autre infirmière (1.8), et quelques tours plus tard (1.19) reprise dans une conclusion qui ratifie par une co-construction à trois (INF1, INF2, EDUC) la représentation commune d'un usager à mobilité sociale limitée de, via une référence à sa mobilité concrète (physique) : il patauge...

La semaine suivante, le cas est débattu à nouveau. L'attribut du déplacement en taxi organisé par la structure (déjà élaboré la semaine précédente, cf. extrait PE350b_claquettes ci-dessus) thématise le manque d'autonomie de l'usager, qui contraste avec ses allées et venues en ville, très autonomes celles-ci. Un nouveau récit, initié par le cadre (AD) (1.18 : et puis lui il vient en ville) qui généralise une observation en instance, réélabore le manque d'engagement de l'usager dans le projet de soin :

\section{2b_taxi (10/12)}

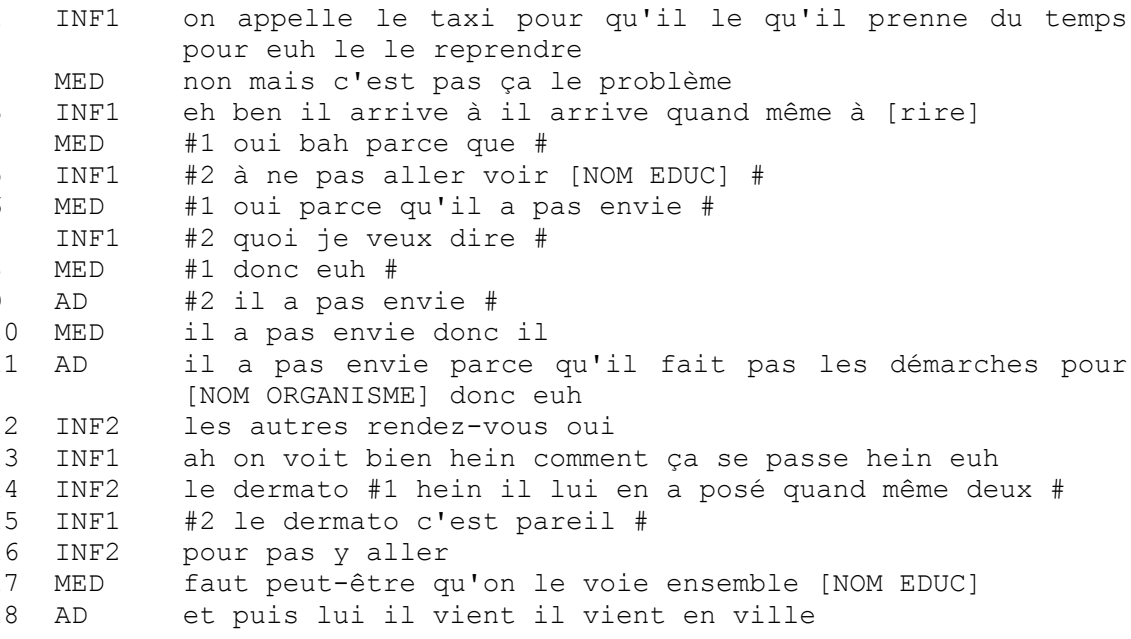




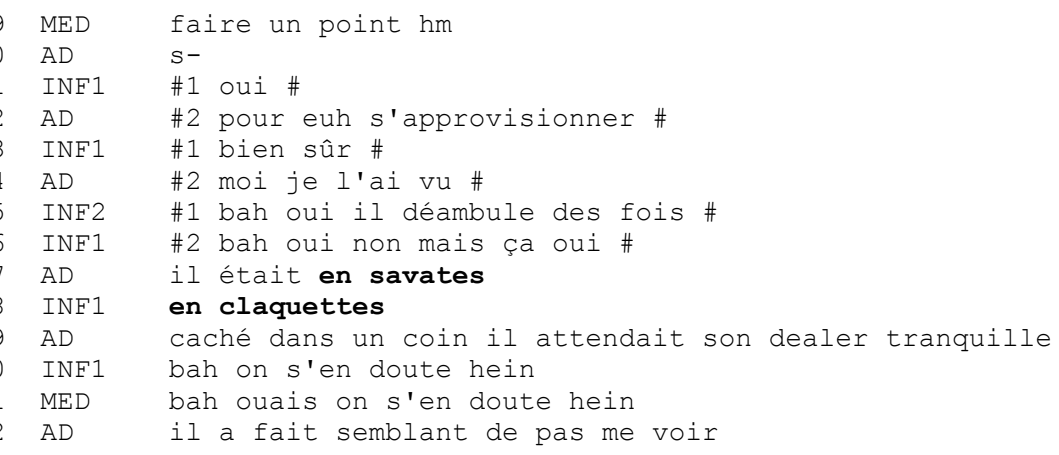

La description de sa tenue, en savates (1.27), rappelle la figure des claquettes, instantanément remobilisée par INF1 (1.28). La reformulation des propos du cadre (AD) par l'infirmière, rare en ce qu'elle pourrait sousentendre une contestation, menaçant l'autorité du cadre, peut s'expliquer par le caractère emblématique de la figure, mobilisée à des fins principalement humoristiques.

Enfin, l'éducateur convoque Fabrice pour démêler les fils de la situation (PE333). Dans une progression très accidentée et avec des reformulations successives de l'attribut (travail, 1.3 - trafic, 1.6 - loisir, 1.11 - activité, 1.12), l'usager formule sa motivation à trouver un travail. L'éducateur demande confirmation de ce qu'il croit comprendre : l'idée du trafic de drogue est encore présente dans l'esprit de l'usager :

\section{PE333_trafic (12/12)}

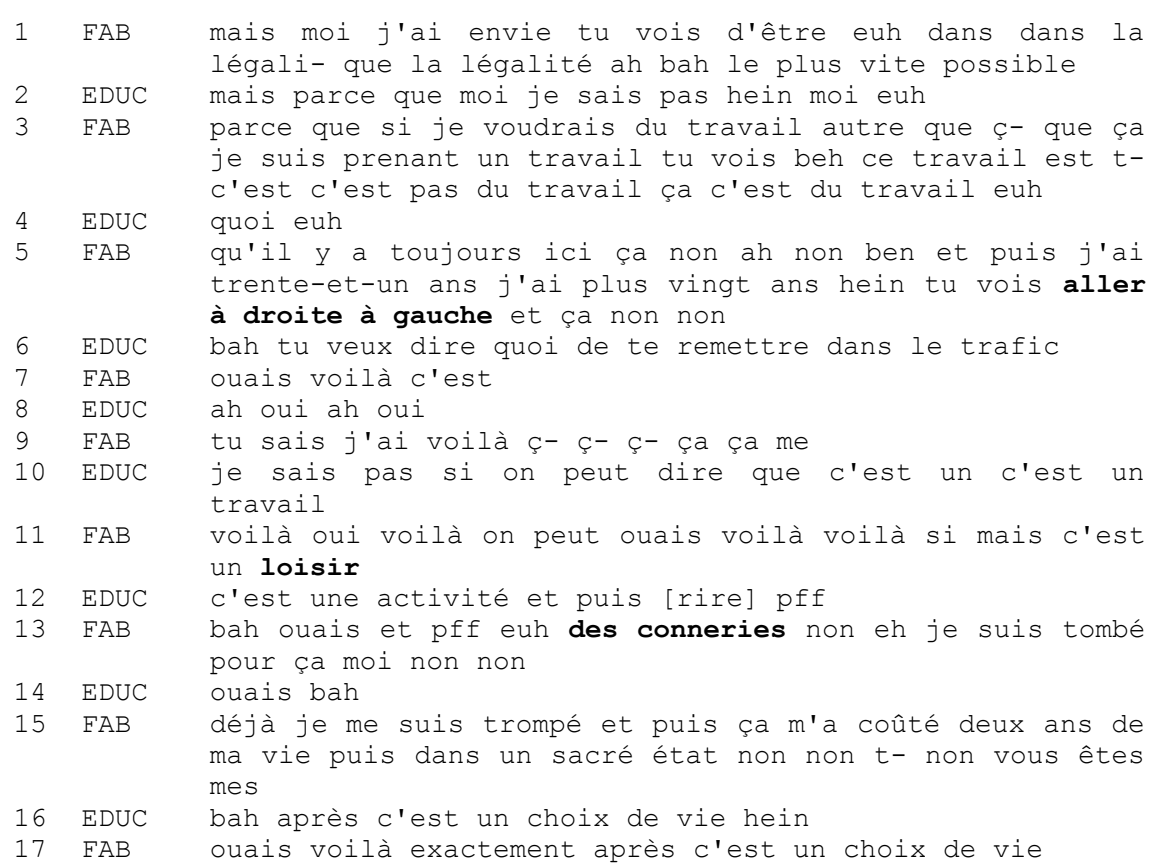




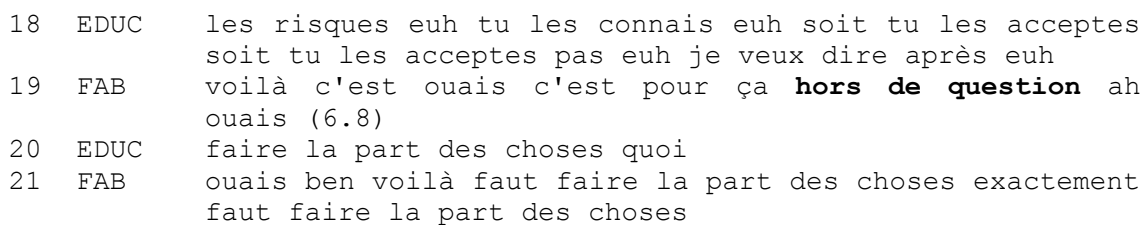

Ici, ce sont les reformulations de l'usager à propos du trafic qui rappellent la figure des claquettes (aller à droite à gauche, 1.5 ; c'est un loisir, 1.11). Celles de l'éducateur se bornent à contester en premier lieu la qualification du trafic comme travail. L'énonciation de l'attribut trafic, entaché d'illégalité et disqualifiant les intentions de l'usager, est suivie par une séquence de réparation (c'est un choix de vie, il faut faire la part des choses, 1.20/21) qui succède à la formulation de l'attribut activité (1.12), élaboration discursive d'un point de vue plus mesuré qui permet de ménager la face de Fabrice, qui ratifie l'ensemble des propos (et le point de vue) du professionnel par des répétitions et marque ainsi sa collaboration.

Le profil de l'usager élaboré par l'équipe, qui voit Fabrice comme coopératif mais peu engagé, est corroboré par le comportement interactionnel de l'usager lui-même, acteur indécis et sans initiative. L'étayage collectif de ce profil marque le rapport de places dans l'interaction par les modalités de la co-construction. De cette coélaboration dans une temporalité médiane émerge un attribut qui consolide la représentation de l'usager élaborée par le groupe des professionnel.les : en claquettes. En effet, la remobilisation de cette figure crée une "résonance" dans l'équipe et le recours à l'humour en permet la condensation comme emblème.

\section{CONCLUSIONS}

Autour du profil de l'usager.e, représenté de manière singulière par chacun des interactant.es, les figures langagières telles en claquettes ou triche à la crapette constituent des attributs construits collectivement en interaction et sont remobilisés dans le but de construire et réévaluer le projet de soin de l'usager.e tout au long du parcours.

L'observation de l'émergence des figures dans différentes temporalités a permis d'en identifier deux procédés majeurs : l'expansion et la condensation. Émergeant en tout début du parcours de soin, alors que le profil de l'usagère est en pleine construction et son projet de soin à l'état d'ébauche, la figure de la crapette permet l'expansion de ce profil dans la négociation interactionnelle. La figure des claquettes intervient, au contraire, à la suite d'un parcours de soin conséquent dans un contexte de consensus préalablement établi, et remis en perspective dans une actualité 
nouvelle ; la figure permet ici la condensation d'un attribut et semble présenter un vecteur emblématique pour l'équipe. Indexicales, ces figures participent à l'élaboration du profil de l'usager.e et constituent, à ce titre, une véritable ressource verbale du soin, qui fait apparaître la qualité de la verbalisation des données relatives à l'usager.e comme l'un des garants du soin.

Pour la recherche en linguistique interactionnelle, ces figures constituent alors un objet d'étude de choix, qui mérite une exploration plus ample pour en établir les procédés argumentatifs spécifiques (p.ex. discours animé, humour), et, dans une approche outillée, les ressources formelles (p.ex. prosodie, rire). La mise en perspective d'une telle analyse des pratiques langagières au travail pourra conduire au développement de modules de formation professionnelle, en sensibilisant les acteurs à la pertinence du travail conversationnel pour le soin. On peut remarquer enfin que les procédés linguistiques mis en exergue dans cette étude nécessitent un cadre temporel suffisamment ample pour en permettre le déploiement, ce qui peut s'avérer contrevenir au paramétrage des actes de soin tel qu'il est pratiqué par l'ARS ${ }^{11}$ à travers les référentiels de rigueur.

Katja Ploog, Université d'Orléans et LLL UMR 7270 CNRS

Caroline Cance, Université d'Orléans et LLL UMR 7270 CNRS

Flora Badin, LLL UMR 7270 CNRS

Camille Noûs, Laboratoire Cogitamus

\section{RÉFÉRENCES BIBLIOGRAPHIQUES}

CANCE, C. PLOOG, K. 2020. « La co-construction discursive des objets de soin en addictologie : du protocole de soin en unité méthadone à la santé globale », Langage et société 2020/1 ( $\left.N^{\circ} 169\right)$, pp. 103-128.

DAUSENDSCHÖN, U. GÜLICH, E. \& KRAFFT, U. 2007. " Vorgeformtheit als Ressource im konversationellen Formulierungsund Verständigungsprozess ", in Hausendorf, Heiko (ed.), Gespräch als Prozess. Linguistische Aspekte der Zeitlichkeit vergbaler Interaktion, Tübingen, Narr, pp. 181-219.

GAJO, L. (ed.). 2004. « Langue de l'hôpital, pratiques communicatives et pratiques de soin ». Cahiers de l'ILSL 16.

GARRIC, N. HERBLAND, A. (ed.) 2020. « Nouveaux discours de la santé et soin relationnel », Langage et société 2020/1 ( $\left.N^{\circ} 169\right)$, Éditions de la Maison des sciences de l'homme, 218 pages.

11 Agence Régionale de Santé. 
GÜLICH, E. 2012. "Conversation analysis as a new approach to the differential diagnosis of epileptic and non-epileptic seizure disorders ", in M. Egbert \& A. Deppermann (eds.), Hearing Aids communication. Integrating social interaction, audiology and user centered design to improve communication with hearing loss and hearing technologies, Mannheim, Verlag für Gesprächsforschung, pp. 146-158.

GÜLICH, E. MONDADA, L. 2001 «Analyse conversationnelle », in Holtus, G., Metzeltin, M., et Schmitt, C. (éd.), Lexikon der romanistischen Linguistik, Tübingen: Niemeyer, Band I,2, pp. 196250.

HERITAGE, J. MAYNARD, D.W. (ed.). 2006. Communication in Medical Care. Interaction Between Primary Care Physicians and Patients, Cambridge, Cambridge University Press.

LANGACKER, R. 1986. Foundations of cognitive grammar, Stanford, Stanford University Press.

PIOT, T. 2018, " Le rôle déterminant et méconnu des conversations soignant-patient au cœur des interactions de soin », Les dossiers des sciences de l'éducation 39, pp. 13-31.

PLOOG, K. EQUOY-HUTIN, S. \& MARIANI-ROUSSET, S. 2014. Parole émergée. Corpus d'interactions de soin en structure pluridisciplinaire. Besançon: Université de Franche-Comté. Métadonnées publiées sur https://cocoon.huma-num.fr/exist/crdo.

PLOOG, K. MARIANI-ROUSSET, S. \& EQUOY-HUTIN (ed.). 2018. Emmêler et démêler la parole. Approche pluridisciplinaire de la relation de soin. Annales Littéraires de l'Université de Franche-Comté, (série Linguistique-Sémiotique-Communication).

SOULET, M.-Y. 2014, « Les raisons d'un succès. La vulnérabilité comme analyseur des problèmes sociaux contemporains », dans BrodiezDolino A. Et al. (dir.), Vulnérabilités sanitaires et sociales. De l'histoire à la sociologie, Rennes, PUR, pp. 59-64.

TRAVERSO, V. 2017. « Formulations, reformulations et traductions dans l'interaction: le cas de consultations médicales avec des migrants $»$. Revue française de linguistique appliquée 2017/2 (Vol. XXII), pp. 147164. 